\title{
A New Method for Cerebral Arterial Stiffness by Measuring Pulse Wave Velocity Using Transcranial Doppler
}

\author{
Xian Fu ${ }^{1}$, Chuming Huang ${ }^{2}$, Ka Sing Wong ${ }^{3}$, Xiangyan Chen ${ }^{3}$ and Qingchun Gao ${ }^{1}$ \\ Xian Fu and Chuming Huang contributed equally to this work \\ ${ }^{1}$ Institute of Neuroscience, the Second Affiliated Hospital of Guangzhou Medical University, Guangzhou, China \\ ${ }^{2}$ Department of Neurology, Shantou Central Hospital, Shantou, China \\ ${ }^{3}$ Department of Medicine and Therapeutics, Prince of Wales Hospital, Chinese University of Hong Kong, Hong Kong, China
}

\begin{abstract}
Aim: Pulse wave velocity (PWV) has been regarded as the "gold standard" measurement of arterial stiffness (AS), but it is still only used in the assessment of central and peripheral arteries. We constructed a new method to evaluate cerebral AS by measuring PWV using transcranial Doppler (TCD). Methods: In all, 90 healthy subjects who received annual health screening were consecutively enrolled in this study between January 2011 and June 2013. Data on clinical characteristics, brachium-ankle (ba) PWV, and carotid-cerebral (cc) PWV measured with our newly constructed method by two experienced operators were recorded. cc PWV was calculated as the distance between two points in the common carotid artery and proximal part of ipsilateral middle cerebral artery, which was divided by the pulse transit time between these two points where the pulse was measured using TCD.

Results: The value of cc PWV was $499.3 \pm 78.6 \mathrm{~cm} / \mathrm{s}$. Correlation between cc PWV and ba PWV in the assessment of AS was $r=0.794(P<0.001)$. The concordance between both the above mentioned methods was good. Interobserver and intraobserver reliability using interclass correlation for measuring cc PWV were $0.815(P<0.001)$ and $0.939(P<0.001)$, respectively. In multivariable analysis, older age $(\beta=4.51, P<0.001)$ and increased diastolic blood pressure $(\beta=2.39, P<0.001)$ were independently associated with higher cc PWV.

Conclusion: cc PWV measured using TCD may be a promising method for the assessment of human cerebral AS, which is independently associated with age and diastolic blood pressure.
\end{abstract}

Key words: Arterial stiffness, Carotid-cerebral pulse wave velocity, Transcranial Doppler

\section{Introduction}

Arterial stiffness (AS) is defined by a reduction in arterial compliance, which has been associated with cardiovascular events and atherosclerotic disease in several reports ${ }^{1-5}$. Clinically, the most representative and noninvasive technique for assessing AS is pulse wave velocity (PWV) measurement ${ }^{6}$. Among PWV measurements, brachial-ankle (ba) PWV has been most widely used as an index of large artery compli-

Address for correspondence: Qingchun Gao, Institute of Neuroscience, the Second Affiliated Hospital, Guangzhou Medical University, 250\# Changgang East Road, GuangZhou, 510260, China

E-mail: qcgao@263.net

Received: October 7, 2015

Accepted for publication: March 6, 2016 ance in previous clinical studies ${ }^{3-5,7,8)}$. ba PWV showing a close correlation with carotid-femoral pulse wave velocity reflects the stiffness of both central and peripheral arteries ${ }^{9)}$. Recent studies have also demonstrated that ba PWV is closely associated with intracranial cerebral atherosclerosis or cerebrovascular disease $^{6,10)}$. However, ba PWV and other PWV measurements cannot be used to directly assess cerebral AS. Approximately two decades ago, Giller et al. ${ }^{11)}$ attempted to estimate PWV in the human cerebral circulation using transcranial Doppler (TCD), but they failed to obtain an accurate value. Therefore, we aimed to construct a novel method including original time and distance assessment systems to directly evaluate human carotid-cerebral (cc) PWV. If this method was applied in clinical practice or general population screening, it would be useful for the further study of 
cerebral atherosclerotic disease.

\section{Aim}

The aim of the present study was to construct a novel method to directly evaluate human cerebral AS based on cc PWV and discuss its determinants in healthy volunteers, which can provide data for further study in clinical practice.

\section{Materials and Methods}

\section{Study Subjects}

Between January 2011 and June 2013, 156 subjects (aged $>18$ years) who received annual health screening in the Second Affiliated Hospital of Guangzhou Medical University, Guangzhou, China were observed. Of them, 90 healthy subjects were consecutively enrolled in this study. The others were excluded for the following reasons: (1) abnormal results as shown in the physical examination, blood analysis, or TCD test during annual health screening; (2) medical histories including hypertension, diabetes, hyperlipidemia, coronary artery disease, cerebrovascular disease, or renal insufficiency; and (3) unsuitable temporal window for conducting TCD monitoring. Baseline information on demographics and medical history was obtained from face-to-face interviews or medical records. Explanatory variables included cardiovascular risk factors, such as current cigarette smoking and regular alcohol consumption, and clinical features. Clinical features in annual health screening included blood pressure (BP; systolic and diastolic BP), heart rate, body mass index, fasting blood glucose, triglyceride, total cholesterol, low and high density lipoprotein, ba PWV, and cc PWV. Written informed consent was obtained from all subjects prior to participation. The study was approved by the ethics committees of the Second Affiliated Hospital of Guangzhou Medical University (Guangzhou). Good Clinical Practice guidelines in accordance with the Declaration of Helsinki were used, and the privacy of patients was strictly protected.

\section{Measurement of cc PWV}

cc PWV was measured with two-channel TCD (EMA-9A; Shenzhen Delicate Electronics Co., Ltd, Shenzhen, China) using $2 \mathrm{MHz}$ and $4 \mathrm{MHz}$ ultrasound transducers in the supine position by two experienced operators. The $2-\mathrm{MHz}$ probe was held to a temporal window by the head frame, which can keep the transducer detecting the proximal part of middle cerebral artery (MCA) in a stable status, and MCA was insonated at a depth of 50-55 $\mathrm{mm}$ using standard criteria ${ }^{12,13)}$. The other $4-\mathrm{MHz}$ transducer in the angle fix- ator was placed on the ipsilateral pulsation point of common carotid artery (CCA) beside the thyroid notch of the neck to detect CCA. The angle fixator can fix the $4-\mathrm{MHz}$ transducer on the neck and regulate its angle from $0^{\circ}$ to $90^{\circ}$ to detect CCA. CCA was insonated at a depth of $40-50 \mathrm{~mm}$. The TCD machine used in this study has a built-in program model called arterial pulse wave detecting system. This system can derive, store, and process signals obtained from transducers on CCA and MCA sites and simultaneously display these signals with expanded waveforms (Fig. $\mathbf{1}$ and $\mathbf{2}$ ).

The explicit arterial pulse waveforms were selected for data analysis using the built-in software of TCD. The selection method of the onset point of the waveform is shown in Fig. 2. First, we defined the root (a) and top (b) points in the ascending stage of the waveform. We use the built-in software to compare values (blood flow velocity) of data points of the waveform in one cardiac cycle and select the data point with minimum and maximum values as the root (a) and top (b) points, respectively. When the waveform had two or three data points, which had the same minimum value in one cardiac cycle, we determined the root point as follows: if the waveform has two data points with the same minimum value, we selected the first data point as the root (a) point; if the waveform has three data points with the same minimum value, we selected the second data point as the root (a) point. Therefore, in one cardiac cycle, each waveform has only one root (a) point. These rules were all strictly followed in this study. Second, we selected point c between points $\mathrm{a}$ and $\mathrm{b}$ in the waveform, which had the maximum vertical distance (red line cc in Fig. 2) to the line connecting the root and top points (line $\mathrm{ab}$ in Fig. 2), and defined point $\mathrm{c}$ as the onset point of this cardiac cycle (Fig. 2). We marked the onset points of CCA and MCA waveforms in one cardiac cycle, and their corresponding time could be obtained in the display (Fig. 2 and 3) ${ }^{14)}$. The time interval between the two onset points was defined as the transit time $(\Delta t$, $\mathrm{ms}$ ) of pulse wave traveled between the two insonation sites (Fig. 3). The mean transit time was then determined from 10 consecutive cardiac cycles. The distance $(\mathrm{D}, \mathrm{cm})$ traveled by the pulse wave in this study was defined as the body surface distance between the two probes using a tape measure plus the cosine of detecting depth for CCA (Fig. 1). Thus, cc PWV calculated from $\mathrm{D}$ traveled by the pulse wave and mean time delay $(\Delta \mathrm{mt})$ as follows: $\mathrm{cc} P W V=\mathrm{D} / \Delta \mathrm{mt}(\mathrm{cm} / \mathrm{s})^{3,15)}$.

\section{Measurement of ba PWV ${ }^{6}$}

Bilateral ba PWV was measured using an automated device (VP-1000; Colin Co. Ltd., Komaki, Japan). Prior to the measurement of ba PWV, subjects 


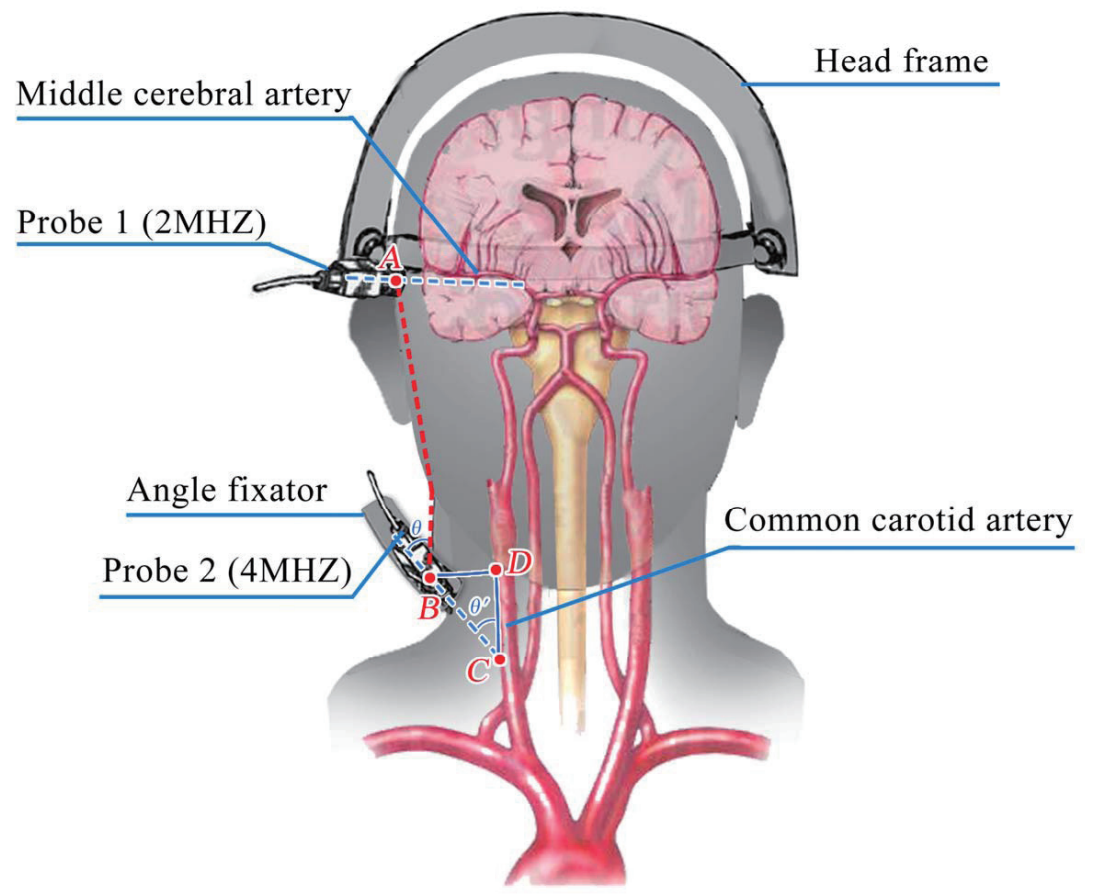

Fig. 1. The method of measuring the distance traveled by the pulse wave

The 2-MHz probe placed in the head frame was held to a temporal window to detect the proximal part of MCA, and the other 4-MHz transducer was placed on the ipsilateral pulsation point of CCA beside thyroid cartilage to detect CCA in an angle fixator which can fix the transducer on the neck and regulate its angle from $0^{\circ}$ to $90^{\circ}$. The distance traveled by the pulse wave was defined as the body surface distance $\left(\mathrm{D}_{\mathrm{AB}}\right)$ between the two probes plus $\cos \theta^{\circ}$ (here, we presumed $\theta \approx \theta^{\prime}$ ) of detected depth $\left(\mathrm{D}_{\mathrm{BC}}\right)$ of CCA. This is $\mathrm{D}(\mathrm{cm}) \approx \mathrm{D}_{\mathrm{AB}}+\mathrm{D}_{\mathrm{CD}}=\mathrm{D}_{\mathrm{AB}}+\mathrm{D}_{\mathrm{BC}}{ }^{*} \cos \theta^{\circ}$. Abbreviations: MCA, middle cerebral artery; CCA, common carotid artery.

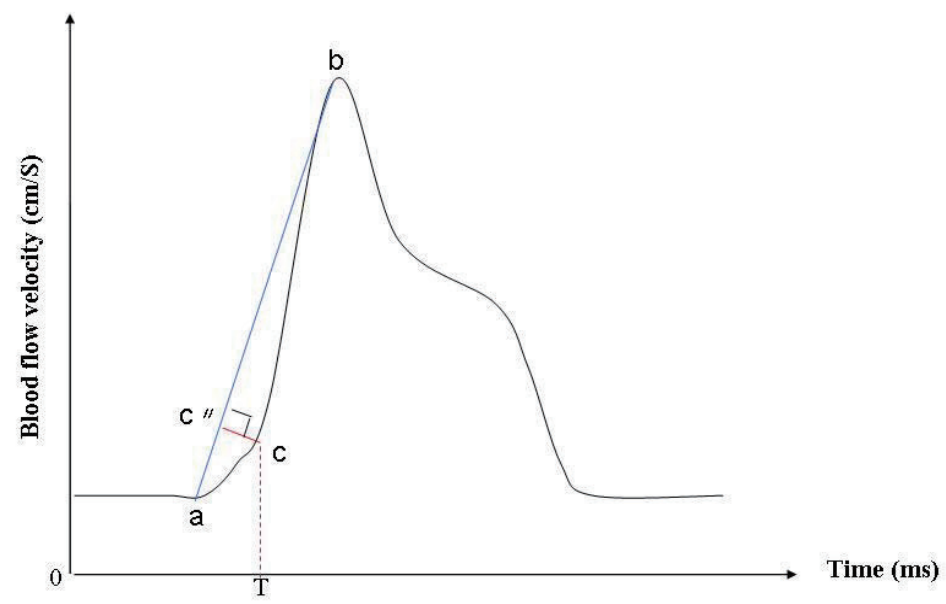

Fig. 2. Description of how to select the onset point in the waveform and determine its corresponding time

First, we defined the root (a) and top (b) points in the ascending stage of the waveform. We use the built-in software to compare the values of data points of the waveform in one cardiac cycle and select the data points with minimum and maximum values as the root (a) and top (b) points. Second, we selected the point $\mathrm{c}$ between points $\mathrm{a}$ and $\mathrm{b}$ on the waveform, which had the maximum vertical distance (red line cc") to the line connecting the root and top points (line ab), and defined point $c$ as the onset point of this cardiac cycle. The corresponding time $(T)$ of the onset point (c) could be obtained in the time coordinates. 


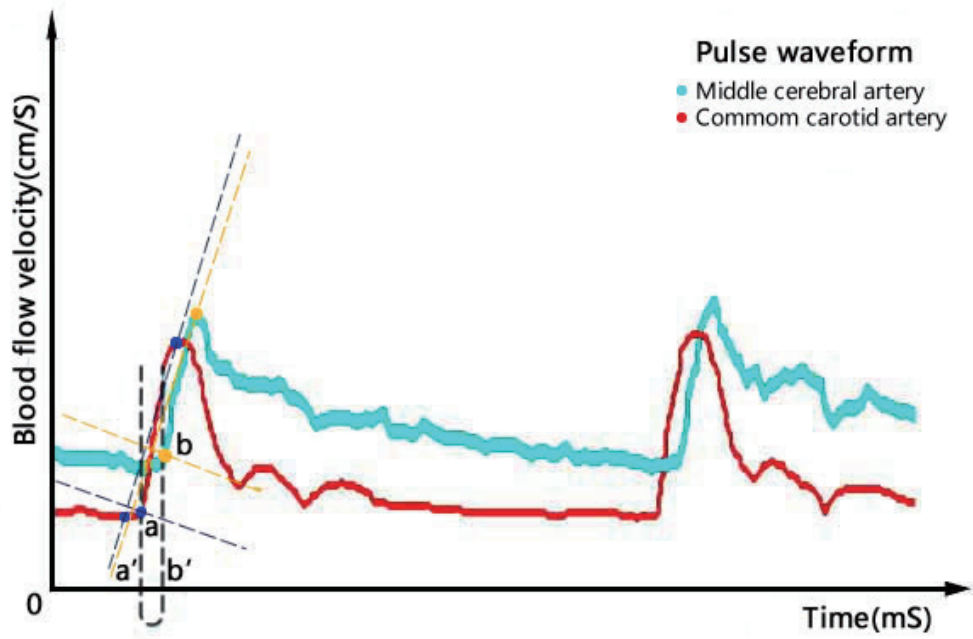

Fig. 3. The method of measurement of transit time of pulse wave between two insonation sites

We marked the onset points $(\mathrm{a}, \mathrm{b})$ of the flow pulses in the two waveforms of CCA and MCA, and the corresponding time ( $\mathrm{Ta}^{\prime}$ and $\mathrm{Tb}^{\prime}$ ) of each onset point could be obtained in the time coordinates. Therefore, $\Delta \mathrm{tab}(\mathrm{ms})=\mathrm{Tb}^{\prime}-\mathrm{Ta}^{\prime}$.

were advised to have a 15-min rest. They were examined in the supine position. This device simultaneously measures bilateral brachial and posterior tibial arterial pulse waveforms and arterial BP using the oscillometric method. Transmission time was calculated as the time taken for the waveform to travel between the right brachium and ankle. Transmission distance between the right brachium and ankle was automatically calculated from patient height.ba PWVs on each side were automatically calculated as PWV $=$ transmission distance/ transmission time. The average values of ba PWVs bilaterally obtained were used for the analysis.

\section{Interobserver and Intraobserver Reliability}

Data were analyzed using the interclass correlation (ICC) coefficient, described in detail in the Statistical Analysis section below to determine the interobserver and intraobserver reliability for cc PWV measurement using TCD as per all the records measured by the two operators.

\section{Statistical Analysis}

Values are reported as the mean \pm standard deviation or number (\%) of subjects. cc PWV and ba PWV were assessed using correlation coefficients and the Bland-Altman method. Simple linear regression analysis was first conducted for factors associated with cc PWV, followed by multiple regression analysis adjusted for significant $(P<0.05)$ variables from the simple linear regression analysis. Statistical significance was established at $P<0.05$. The ICC coefficient was used as an index of interobserver and intraobserver reliability/agreement ${ }^{16)}$. The interobserver and intraobserver reliability were assessed by fitting two-way mixed effects model using the reliability analysis procedure in SPSS, where the value of cc PWV was modeled with operator and subject entered as random effects. The clinical significance of ICC coefficients was interpreted as excellent, ICC $\geq 0.80$; good, $0.60 \leq \mathrm{ICC}<0.80$; moderate, $0.40 \leq \mathrm{ICC}<0.60$; and poor, ICC $<0.40$. Statistical analyses were performed using the SPSS software for Windows, version 13.0 (SPSS Inc, Chicago, IL, USA).

\section{Results}

\section{Baseline Characteristics}

In all, 90 subjects (male, 46; female, 44) who met the criteria were enrolled in this study. The mean age was $46 \pm 11.7$ years. The values of cc PWV and ba PWV were $499.3 \pm 78.6 \mathrm{~cm} / \mathrm{s}$ and $1296.5 \pm 206.1 \mathrm{~cm} /$ $s$, respectively. Other baseline information on demographics, clinical features, and PWV for primary analysis is presented in Table 1.

\section{Interobserver and Intraobserver Reliability of cc PWV} Interobserver and intraobserver reliability using ICC for measuring cc PWV were $0.815(P<0.001)$ and $0.939(P<0.001)$, respectively.

\section{cc PWV versus ba PWV in the Assessment of AS}

cc PWV measured using TCD correlated with ba PWV in the assessment of AS $(r=0.794, P<0.001$; 
Table 1. Baseline characteristics of study subjects $(N=90)^{*}$

\begin{tabular}{lc}
\hline Category & Variable \\
\hline Age(year) & $46.0 \pm 11.7$ \\
Male & $46(51.1)$ \\
Cigarette smoking & $27(30.0)$ \\
Alcohol consumption & $15(16.7)$ \\
Systolic blood pressure $(\mathrm{mmHg})$ & $118 \pm 17.0$ \\
Diastolic blood pressure $(\mathrm{mmHg})$ & $77.0 \pm 10.3$ \\
Heart rate $($ bpm) & $68.0 \pm 11.0$ \\
Body mass index (kg/m $\left.{ }^{2}\right)$ & $23.0 \pm 3.2$ \\
Fasting blood glucose $(\mathrm{mmol} / \mathrm{L})$ & $5.0 \pm 0.7$ \\
Triglyceride (mmol/L) & $1.4 \pm 1.5$ \\
Total cholesterol (mmol/L) & $4.8 \pm 1.3$ \\
Low density lipoprotein $(\mathrm{mmol} / \mathrm{L})$ & $2.9 \pm 1.2$ \\
High density lipoprotein $(\mathrm{mmol} / \mathrm{L})$ & $1.4 \pm 0.3$ \\
Distance traveled by cc PWV(cm) & $17.7 \pm 1.1$ \\
Brachium-ankle pulse wave velocity $(\mathrm{cm} / \mathrm{s})$ & $1296.5 \pm 206.1$ \\
Carotid-cerebral pulse wave velocity $(\mathrm{cm} / \mathrm{s})$ & $499.3 \pm 78.6$ \\
\hline
\end{tabular}

*Values are presented as $n(\%)$ or mean \pm standard deviation.

A

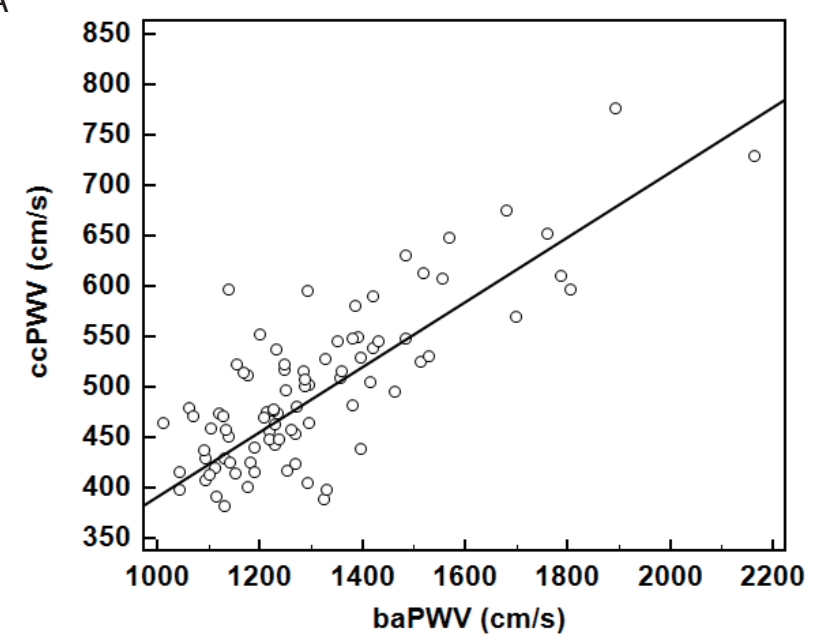

B

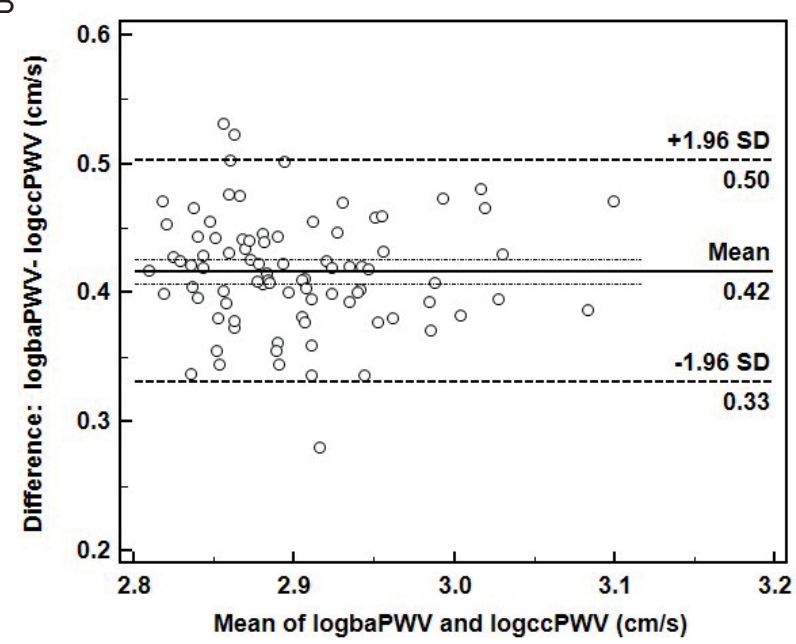

Fig. 4.

A: The relationship between cc PWV and ba PWV was best described by a linear mode $(r=0.794, P<0.001)$. B: Scatterplots showed the average of $\log \mathrm{cc}$ PWV and $\log$ ba PWV $(\mathrm{cm} / \mathrm{s}$, $\mathrm{x}$ axis) and the difference between log ba PWV and log cc PWV. The mean difference, limits of agreement (LOC), and $95 \%$ CI of LOC between methods are shown.

Abbreviations: cc PWV, carotid-cerebral pulse wave velocity; ba PWV, brachium-ankle pulse wave velocity.

Fig. 4A). The Bland-Altman plot (Fig.4B) confirmed that the agreement between both methods in the assessment of AS was good, and it was high at 95\% confidence intervals (CI) of the lower and upper boundaries of limits of agreement.

\section{Determinants of cc PWV}

In this study, factors associated with cc PWV in simple linear regression analysis were age $(P<0.001)$, systolic and diastolic BP $(P<0.001)$, and cigarette smoking $(P=0.019)$, and in multiple linear regression analysis adjusted for significant $(P<0.05)$ variables from the simple linear regression analysis, they were age, cigarette smoking, and diastolic BP. Older age $(\beta$ $=4.51, P<0.001)$ and increased diastolic $\mathrm{BP}(\beta$ $=2.39, P<0.001$ ) were independently associated with higher cc PWV (Table 2). 
Table 2. Associations between Carotid-cerebral pulse wave velocity and subject characteristics $(N=90)$

\begin{tabular}{|c|c|c|c|c|c|c|}
\hline \multirow[t]{3}{*}{ Characteristic } & \multicolumn{3}{|c|}{ Univariate } & \multicolumn{3}{|c|}{ Multivariate $^{\dagger}$} \\
\hline & $\begin{array}{l}\text { Parameter } \\
\text { estimate }\end{array}$ & Standard error & $p$ Value & $\begin{array}{c}\text { Parameter } \\
\text { estimate }\end{array}$ & Standard error & $p$ Value \\
\hline & $(\beta)$ & & & $(\beta)$ & & \\
\hline Age(years) & 5.32 & 0.45 & $<0.001$ & 4.51 & 0.43 & $<0.001$ \\
\hline Male & -18.64 & 16.95 & 0.274 & - & - & - \\
\hline Cigarette smoking & 42.60 & 17.77 & 0.019 & - & - & - \\
\hline Alcohol consumption & 26.10 & 22.28 & 0.245 & - & - & - \\
\hline Systolic blood pressure (mmHg) & 2.65 & 0.41 & $<0.001$ & - & - & - \\
\hline Diastolic blood pressure $(\mathrm{mmHg})$ & 4.37 & 0.69 & $<0.001$ & 2.39 & 0.49 & $<0.001$ \\
\hline Heart rate(bmp) & -0.96 & 0.78 & 0.224 & - & - & - \\
\hline Body mass index $\left(\mathrm{kg} / \mathrm{m}^{2}\right)$ & 0.51 & 2.63 & 0.848 & - & - & - \\
\hline Fasting blood glucose (mmol/l) & 2.66 & 12.71 & 0.835 & - & - & - \\
\hline Triglyceride $(\mathrm{mmol} / \mathrm{l})$ & 2.16 & 5.56 & 0.699 & - & - & - \\
\hline Total cholesterol (mmol/l) & 6.33 & 6.64 & 0.343 & - & - & - \\
\hline Low density lipoprotein $(\mathrm{mmol} / \mathrm{l})$ & -4.06 & 9.98 & 0.687 & - & - & - \\
\hline High density lipoprotein $(\mathrm{mmol} / \mathrm{l})$ & -20.31 & 30.61 & 0.509 & - & - & - \\
\hline
\end{tabular}

Adjusted for significant $(p<0.05)$ variables from the simple linear regression analysis: Age, cigarette smoking, and diastolic blood pressure.

\section{Discussion}

To the best of our knowledge, this was the first accurate assessment of human cerebral AS by measuring PWV. First, we originally constructed a time assessment system to calculate transit times of pulse waves and a distance assessment system to accurately measure the distance between the two insonation sites. These two systems can make the values of transient time and distance approach the real value and decrease the deviation in this study. Second, an angle fixator with an adjustable angle was used in this study. The fixator can keep the 4-MHz probe motionless on the neck and simultaneously regulate the angle to detect the pulse wave of CCA. Furthermore, we utilized a head frame to fix the $2-\mathrm{MHz}$ transducer on the temporal window for the detection of MCA in a stable status. All the abovementioned methods were followed to ensure accurate measurement of cc PWV. Two decades ago, Giller et al. ${ }^{11)}$ tried to estimate PWV in the human cerebral circulation using TCD, but they failed to obtain the accurate value of PWV. First, they regarded the transmission distance as $10 \mathrm{~cm}$ in all subjects; however, the distance determined by individual skull characteristics and intracranial artery shape varies in different individuals and races. In this study, we found that the distances in all subjects were over 15 $\mathrm{cm}$ using our method of measurement. Second, they marked the onset point of the waveform at the intersection of the straight lines fitting the slope of the waveform before and after the time of rise ${ }^{11)}$. This method was crude and decreased the accuracy of the pulse waveform transient time measurement. As such, the measurement of cc PWV in this study was the first accurate assessment of human cerebral AS using TCD.

This method of human AS assessment with cC PWV using TCD is a valid and noninvasive approach. ba PWV is widely used to estimate stiffness in both central and peripheral arteries in clinical practice ${ }^{6,7,9,10)}$. Numerous studies have demonstrated that ba PWV is closely associated with intracranial atherosclerosis or cerebrovascular disease $^{6,10)}$. In this study, we compared cc PWV and ba PWV in the assessment of AS and found that both methods had good correlation and concordance in healthy human volunteers, although the values of cc PWV and ba PWV were different. We believe the different characteristics of artery anatomic structure may be the main reason for the different values between cc PWV and ba PWV in normal subjects, and the underestimation of distances traveled was also involved. In addition, TCD is a noninvasive tool and has portable and relatively inexpensive characteristics, which makes it possible for this method to be used not only for clinical practice but also for screening purposes. On the other hand, we found that older age and higher diastolic BP were significantly associated with cc PWV in healthy human volunteers in this study. These results were consistent with previous findings that showed an association of increased AS with older age and higher $\mathrm{BP}^{7,9,17)}$.

The present study has several limitations. First, this study defined the distance traveled by pulse wave 
as the body surface distance between the two probes plus cosine of detecting depth for CCA. However, the body surface distance cannot accurately represent the distance of the corresponding artery because the terminal internal carotid artery is circuitous. Second, the subjects in this study were all healthy volunteers. In the present study, we aimed to validate the method for evaluating the human cerebral AS and discuss the normal value of cc PWV and its determinants in healthy volunteers. However, this was just the first step; further study should focus on applying this method in clinical practice with a large sample size. On the other hand, we did not use magnetic resonance imaging (MRI) to evaluate subjects' brains. Therefore, some subjects with asymptomatic brain diseases may not be excluded in this study. Third, all cc PWV measurements were independently executed by two experienced operators in this study; therefore, only one ICC coefficient was obtained in the present study.

In conclusion, cc PWV may be a promising method for the detection of human cerebral AS, which was independently associated with age and diastolic BP.

\section{Acknowledgements}

This study was funded by Program of National Natural Science Foundation of China (grant No.81371573) and Science and Technology Planning Project of Guangdong Province (Grant No.2012B031800435), China.

\section{Conflict of Interest}

The authors declare no conflict of interest.

\section{References}

1) Laurent S, Cockcroft J, Van Bortel L, Boutouyrie P, Giannattasio C, Hayoz D, Pannier B, Vlachopoulos C, Wilkinson I, Struijker-Boudier H: Expert consensus document on arterial stiffness: methodological issues and clinical applications. Eur Heart J, 2006; 27: 2588-2605

2) Laurent S, Boutouyrie P: Arterial stiffness and stroke in hypertension: therapeutic implications for stroke prevention. CNS Drugs, 2005; 19: 1-11

3) Zoungas S, Asmar RP: Arterial stiffness and cardiovascular outcome. Clin Exp Pharmacol Physiol, 2007; 34: 647-651

4) Lee HS, Kim HL, Kim H, Hwang D, Choi HM, Oh SW, Seo JB, Chung WY, Kim SH, Kim MA, Zo JH: Incremental Prognostic Value of Brachial-Ankle Pulse Wave Velocity to Single-Photon Emission Computed Tomography in
Patients with Suspected Coronary Artery Disease. Journal of atherosclerosis and thrombosis, 2015; 22: 1040-1050

5) Gomez-Sanchez L, Garcia-Ortiz L, Patino-Alonso MC, Recio-Rodriguez JI, Frontera G, Ramos R, Marti R, Agudo-Conde C, Rodriguez-Sanchez E, Maderuelo-Fernandez JA, Gomez-Marcos MA: The Association Between the Cardio-ankle Vascular Index and Other Parameters of Vascular Structure and Function in Caucasian Adults: MARK Study. Journal of atherosclerosis and thrombosis, 2015; 22: 901-911

6) Kim J, Cha MJ, Lee DH, Lee HS, Nam CM, Nam HS, Kim YD, Heo JH: The association between cerebral atherosclerosis and arterial stiffness in acute ischemic stroke. Atherosclerosis, 2011; 219: 887-891

7) Tomiyama $H$, Yamashina A: Non-invasive vascular function tests: their pathophysiological background and clinical application. Circ J, 2010; 74: 24-33

8) Yamashina A, Tomiyama H, Arai T, Hirose K, Koji Y, Hirayama Y, Yamamoto Y, Hori S: Brachial-ankle pulse wave velocity as a marker of atherosclerotic vascular damage and cardiovascular risk. Hypertens Res, 2003; 26: 615-622

9) Lee HY, Oh BH: Aging and arterial stiffness. Circ J, 2010; 74: 2257-2262

10) Kim DH, Kim J, Kim JM, Lee AY: Increased brachialankle pulse wave velocity is independently associated with risk of cerebral ischemic small vessel disease in elderly hypertensive patients. Clin Neurol Neurosurg, 2008; 110: 599-604

11) Giller CA, Aaslid R: Estimates of pulse wave velocity and measurement of pulse transit time in the human cerebral circulation. Ultrasound Med Biol, 1994; 20: 101-105

12) Aaslid R, Markwalder TM, Nornes H: Noninvasive transcranial Doppler ultrasound recording of flow velocity in basal cerebral arteries. J Neurosurg, 1982; 57: 769-774

13) Aaslid R, Huber P, Nornes H: A transcranial Doppler method in the evaluation of cerebrovascular spasm. Neuroradiology, 1986; 28: 11-16

14) Hamilton PK, Lockhart CJ, Quinn CE, McVeigh GE: Arterial stiffness: clinical relevance, measurement and treatment. Clin Sci (Lond), 2007; 113: 157-170

15) Adji A, O’Rourke MF, Namasivayam M: Arterial stiffness, its assessment, prognostic value, and implications for treatment. Am J Hypertens, 2011; 24: 5-17

16) Liel-Cohen N, Tsadok Y, Beeri R, Lysyansky P, Agmon Y, Feinberg MS, Fehske W, Gilon D, Hay I, Kuperstein R, Leitman M, Deutsch L, Rosenmann D, Sagie A, Shimoni $S$, Vaturi M, Friedman Z, Blondheim DS: A new tool for automatic assessment of segmental wall motion based on longitudinal 2D strain: a multicenter study by the Israeli Echocardiography Research Group. Circulation Cardiovascular imaging, 2010; 3: 47-53

17) Oliver JJ, Webb DJ: Noninvasive assessment of arterial stiffness and risk of atherosclerotic events. Arterioscler Thromb Vasc Biol, 2003; 23: 554-566 